die Labor- und Lungenärzte, sie befinden sich durchweg zu $100 \%$ in der Weiterentwicklungsphase. Allerdings sind aber auch gerade die Laborärzte aufgrund ihrer Praxisstruktur und der Tatsache, dass sie Auftragsarbeiten anderer Kollegen übernehmen, häufig schon länger an das QM und eine Zertifizierung gewöhnt. Denn hier ist auch der Qualitätswettbewerb unter Konkurrenten größer, als in anderen ambulanten Bereichen.

Gleicherweise weit fortgeschritten in Sachen QM sind die Augenärzte - immerhin $87 \%$ haben bereits den Prozess der kontinuierlichen Weiterentwicklung eingeläutet; gefolgt von den Pathologen mit $82 \%$, den Nervenärzten mit $80 \%$, den Hautärzten mit $76 \%$ und den Urologen mit $72 \%$.

Die meisten QM-Verweigerer gibt es übrigens bei den Psychotherapeuten, doch auch hier handelt es sich um gerade einmal $3 \%$.

Ebenfalls in die QM-Statistik eingegangen sind MVZ. Auch hier zeigt sich: Mehr als jedes zweite MVZ erfüllt seine QM-Pflicht und hat bereits die Phase der Weiterentwicklung erreicht. Über ein Drittel befindet sich aber auch noch in der Phase der Umsetzung, 12 \% prüfen derzeit ihr QM.

RebekkaHöhl

\author{
Die QM-Stichprobe \\ 2,5\% der Vertragsarztpraxen werden \\ jährlich von den KVen in Sachen QM \\ geprüft. Da die Praxen im Rahmen ei- \\ ner Zufallsstichprobe ausgewählt wer- \\ den, weiß man vorher nie, wen es trifft. \\ Außerdem schicken die KVen keine Prü- \\ fer in die Praxen. Die Ärzte müssen le- \\ diglich anhand eines Fragebogens dar- \\ legen, wie weit sie mit ihrem QM sind. \\ Die komplette QM-Statistik der KBV \\ steht unter: \\ www.g-ba.de/informationen/ \\ beschluesse/1643/
}

\title{
Werbung in eigener Sache meist mit gemischten Gefühlen
}

\section{Die Widersprüchlichkeit in Einstellung und Umgang vieler Ärzte mit dem Thema Praxismarketing ist das prägnante Resultat der Studie „Ärzte im Zukunftsmarkt Gesundheit 2012" der Hamburger Stiftung Gesundheit.}

ᄃ twa die Hälfte der Befragten für die ¿ Studie „Ärzte im Zukunftsmarkt Gesundheit 2012"hält Marketing für wichtig oder sogar sehr wichtig. Doch ein eigenes Budget dafür haben lediglich $14 \%$ der Befragten eingerichtet. Ebenfalls widersprüchlich: Viele Praxisinhaber verzichten wegen rechtlicher Risiken auf eine eigene Website, doch nur jeder elfte befrag- te Arzt mit eigener Website wurde wegen deren Inhalt schon einmal abgemahnt.

An der Online-Befragung nahmen im Herbst vergangenen Jahres 799 Ärzte, Zahnärzte und psychologische Psychotherapeuten teil. Ein Viertel der befragten Niedergelassenen steht Marketing grundsätzlich ablehnend gegenüber, ein weiteres Viertel zeigt sich unentschlos- sen. Die Studienautoren der Gesellschaft für Gesundheitsmarktanalyse (GGMA) kommen zu dem Schluss, dass Ärzte ihre Praxis noch immer eher konservativ führen und eine systematische Kommerzialisierung ablehnen. Laut Studie hat fast jeder zweite niedergelassene Arzt in Deutschland derzeit keine eigene Praxis-Website. Trotzdem halten fast $71 \%$ der Befragten die Internetpräsenz für die wichtigste Marketingmaßnahme, gefolgt vom Praxispersonal (59 \%) und Visitenkarten (45\%).

\section{Bedarfsplanung: Neue Chancen für Fachärzte?}

\section{Seit 1. Januar 2013 ist die neue Bedarfsplanungsrichtlinie in Kraft. Auch für bereits zugelassene Vertragsärzte hat sie Konsequenzen.}

$\mathrm{D}$ ie bisherige Bedarfsplanung sah für alle Arztgruppen einheitliche Planungsbereiche vor, nämlich Kreis, kreisfreie Stadt oder Kreisregion. Im fachärztlichen Bereich werden jetzt jedoch drei Versorgungsebenen unterschieden: -Allgemeine fachärztliche Versorgung (Augenärzte, Chirurgen, Gynäkologen, Dermatologen, HNO-Ärzte, Nervenärzte, Orthopäden, Psychotherapeuten, Urologen, Kinderärzte)

-Spezialisierte fachärztliche Versorgung (Anästhesisten, fachärztlich tätige Internisten, Kinder- und Jugendlichenpsychiater, Radiologen)
_Fachärztliche Versorgungsebene (Humangenetiker, Laborärzte, Neurochirurgen, Nuklearmediziner, Pathologen, Fachärzte für physikalische und rehabilitative Medizin, Strahlentherapeuten, Transfusionsmediziner)

Für Ärzte der allgemeinen fachärztlichen Versorgung bleibt es bei der Planung in kreisfreier Stadt, Landkreis oder Kreisregion. Die spezialisierte fachärztliche Versorgung aber wird künftig auf die Planungsbereiche entsprechend den Raumordnungsregionen des Bundesinstitutes für Bau-, Stadt- und Raumforschung (BBSR) ausgelegt. In diesen 96 Regionen sind mehrere kreisfreie Städte und Landkreise zusammengefasst. Zum Beispiel gehört jetzt Dachau zu demselben Planungsbereich wie München. Theoretisch könnte ein in Dachau niedergelassener fachärztlich tätiger Internist seine Praxis nach München verlegen. Anders herum geht das natürlich auch. Für Ärzte der spezialfachärztlichen Versorgung ergeben sich damit erhebliche Möglichkeiten, neue Standorte durch Verlegung eines Vertragsarztsitzes in weniger gut versorgte Bereiche zu errichten.

Als Planungsbereich für die letzte Versorgungsebene, die gesonderte fachärztliche Versorgung, wurde der Bezirk der jeweiligen $\mathrm{KV}$ festgelegt.

Dr. Ingo Pflugmacher, Fachanwalt für Medizinund Verwaltungsrecht, Partner der Anwaltskanzlei Busse \& Miessen in Bonn 